TITLE:

\title{
Physical Architectures and Mathematical Models for Electric- Power Management of Multiple Homes
}

\author{
$\operatorname{AUTHOR}(\mathrm{S})$ : \\ Susuki, Yoshihiko; Kazaoka, Ryoya; Hikihara, \\ Takashi
}

\section{CITATION:}

Susuki, Yoshihiko ... [et al]. Physical Architectures and Mathematical Models for ElectricPower Management of Multiple Homes. Proceedings of the 50th IEEE Conference on Decision and Control and European Control Conference (CDC-ECC11) 2011: 7703-7710

\section{ISSUE DATE:}

2011-12

URL:

http://hdl.handle.net/2433/151842

\section{RIGHT:}

(c) 2011 IEEE. Personal use of this material is permitted. Permission from IEEE must be obtained for all other uses, in any current or future media, including reprinting/republishing this material for advertising or promotional purposes,

creating new collective works, for resale or redistribution to servers or lists, or reuse of any copyrighted component of this work in other works.; This is not the published version. Please cite only the published version.; この論文は出版社版

でありません。引用の際には出版社版をご確認ご利用ください。 


\title{
Physical Architectures and Mathematical Models for Electric-Power Management of Multiple Homes
}

\author{
Yoshihiko Susuki, Ryoya Kazaoka, Takashi Hikihara
}

\begin{abstract}
We report physical architectures and mathematical models for electric-power management of multiple homes. The notion of home is a unit of small-scale electric power system close to consumers and corresponds to an entity of the physical architecture for electric-power management. The home includes own energy source, energy storage, residential load, power conversion circuits, and control systems. An entire power system consists of multiple homes that are interconnected via a distribution network and are connected to the commercial power grid. By applying a technology of grid-connected inverters for each home, we show that the mathematical model derived for the power system has a coupling structure similar to that in the multi degree-of-freedom swing equations for analysis of multi-machine power systems. Numerical simulations of the model allow us to define nominal dynamics of the power system, in which the supercritical Hopf bifurcation and associated sustained oscillations in frequency and active power are identified under a realistic setting of model parameters.
\end{abstract}

\section{INTRODUCTION}

Background.-Energy distribution has been traditionally managed and regulated by regional power and gas suppliers. However, because of the complex physical dynamics due to multiple scales in time and space, this is a very challenging task, especially on the demand side. Many researchers in science and technology begin intensive efforts to overcome these challenges through the integration of information, communications, energy, power, and control technologies. The integration begins at the smallest grid inside the home, then moves to building units, and finally reaches the local area. There are many ongoing research projects on energy distribution such as the so-called Smart Grid vision in the United States [1]. Thus, it is widely recognized that demand side management is the key enabler to accomplishing the energy distribution in a stable, resilient, and energy-efficient way.

Our Research Project.-Currently, we are developing a methodology and tools for electric-power management of multiple homes, as a part of the research project Integrated Technology of Information, Communications, and Energy [2]. One aim of the project is to develop a new energy technology integrated with the highly-developed ICT (Information and Communications Technology) that achieves a future energyefficient life of consumers without loss of their quality-of-life (QoL). The notion of home used above is a unit of smallscale power system close to consumers and corresponds to an entity of the physical architecture for electric-power

This work is supported in part by NICT Research Project ICE-IT.

The authors are with the Department of Electrical Engineering, Kyoto University, Katsura, Nishikyo, Kyoto 615-8510 Japan (e-mail: susuki@dove.kuee.kyoto-u.ac.jp).

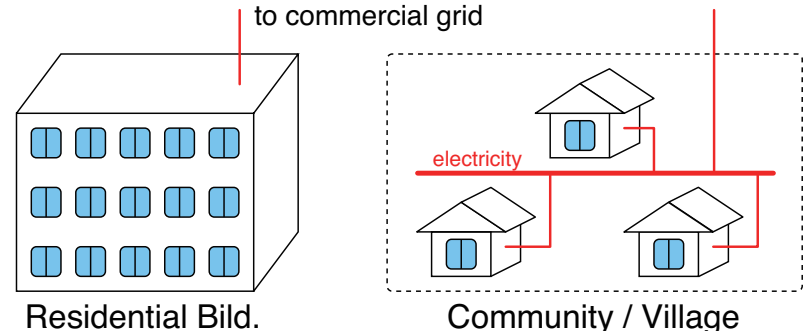

Fig. 1. Practical examples for application of electric-power management of multiple homes: a large residential building with multiple rooms used by different consumers and a community or village with multiple, small residential buildings. The residential building and community are connected to the commercial power grid.

management that we propose in this paper. The home can consist of own energy source, energy storage, residential load, power conversion circuits, and control systems. Examples of the energy source are photovoltaic (PV) array, microcogeneration plant, and fuel cell. Typical examples of the energy storage are the system of Lithium-Ion batteries and electric vehicle connected to a home. An entire power system includes such homes that are interconnected via a distribution network and are connected to the commercial power grid. Practical examples of the power system are shown in Fig. 1 and include a small community with multiple individual houses. In our part of the project, by controlling electricpower flows in one home and between different homes, we intend to achieve a novel electric-power management that does not affect the commercial power grid as well as the QoL. The goal of our part is to validate the methodology and tools in practical situations of residential buildings. This is similar to the development of smart community such as the Amsterdam Smart City [3].

Purpose and Contributions.-The purpose of this paper is to describe the idea, approach, and preliminary results on electric-power management of multiple homes. Within the architecting process for the electric power system, it needs to determine several types of architectures - in the language of the monograph [4], functional, physical, technical, and dynamic operational-and to analyze their integration. In this paper, we address a physical aspect and mathematical modeling for the electric-power management. The contributions of this paper are threefold. First, we introduce physical architectures of the electric power system with multiple homes. A key point of these architectures is to apply the technology of bi-directional, single-phase grid-connected 
inverters that is proposed in [5]-[7] and termed in [7] as the synchronous inverter. This application makes it possible to maintain frequency synchronization, which is a dynamical analogue of synchronous generators, and to enhance stability of the entire power system against a disturbance. We will term the inverter circuit as the interface circuit. Second, we develop a mathematical model to represent slow dynamics of the power system. The slow dynamics correspond to time responses of frequency and active power in the mid-term regime (order of seconds to hours). Due to the design of the interface circuit, we show that the developed model has a coupling structure similar to that in the multi degreeof-freedom swing equations for transient stability analysis of multi-machine power grids [8]-[10]. Third, we present an example of the slow dynamics of the power system in a realistic setting of parameters. The setting is based on commercial residential buildings and power conditioning systems in Japan. Numerical simulations of the mathematical model allow us to define nominal dynamics of the power system, in which the supercritical Hopf bifurcation and associated sustained oscillation in frequency and active power are identified. Also we will indicate that the nominal dynamics may induce a nonlinear propagation of the uncertain input of a renewable energy source to the active power output of each home.

Organization.- - In Section II we introduce physical architectures for electric-power management of multiple homes. In Section III we develop a mathematical model to represent slow dynamics of the power system with $N$ homes. In Section IV we present a preliminary result on numerical simulations of the slow dynamics in the case of three $(N=$ 3) homes. Section V concludes this paper with a summary and future work.

\section{Physical Architectures}

In this section, we introduce control objectives for the electric-power management and describe physical architectures of the electric power system with multiple homes. In the language of [4], the physical architecture is at minimum a node-arc representation of physical resources and their interconnections; also the architecture comprises entities and the structure of relationships and interfaces between them. Following these, we describe an in-home power system as the entity, an inter-home distribution network as the structure of relationships, and an interface circuit as the interface between them.

\section{A. Control Objectives}

Our target level to control electric-power is in the range of one-home to multiple-home capacities, that is, a few $\mathrm{kW}$ to a few dozen $\mathrm{kW}$. In this target level, there are three control objectives that should be achieved in our management. The first objective is to realize new functionalities of electricpower management using the ICT. Examples of them include the delivery of electric-power between different homes and the application of control mechanisms driven by market and energy-efficiency policies. The second one is to maintain stability of the electric power system with multiple homes.

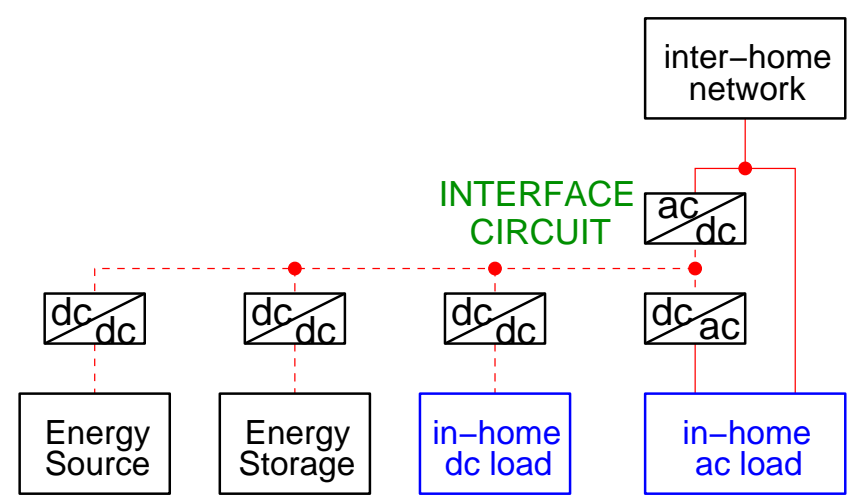

Fig. 2. Physical architecture of the in-home power system. The system consists of own generation unit, storage unit, in-home ac and dc loads, and power conversion circuits. The solid line represents the ac electricity line, and the dashed line the dc electricity line. Power between the inside and outside of home is mainly regulated with the main power conversion circuit which we term as the interface circuit. The interface circuit connects the in-home dc system with the ac one and has an ability of enhancing stability of the connected power system.

Because the notion of stability is very broad [11], it needs to use appropriate definitions for stability of interest. Since the first purpose implies the occurrence of non-static flow in the power system, steady-state and dynamic stability of frequency and voltage are required to be maintained. The third objective is to enhance reliability of the entire power system. This includes the tasks of keeping the quality of electric-power, of reducing the rate of accidents, and of imposing the zero reverse flow to the commercial grid. For the quality task, it is possible to utilize the control ability of grid-connected inverters [12].

Note that these objectives are present for the so-called Microgrid [13], [14]. The Microgrid is being developed as an entity of the conventional power grid and is a unit of small-scale power system with own renewable energy source, storage device, and load. Compared with this, there are several different features of the power system which we address in this paper. First, the target level of our management is lower than that of most Microgrid projects [14]. Second, the spatial area of the target power system is in the range of a few dozen homes and is narrower than these projects. Lastly, we control electric-power at the demand side close to home consumers and contribute total energy-efficiency in the current electricity infrastructure.

\section{B. In-home Architecture}

Figure 2 shows the physical architecture of the in-home power system which we propose in this paper. This consists of an energy generation unit (photovoltaic array, microcogeneration plant, fuel cell, etc.), an energy storage unit (secondary Lithium-Ion battery, electric vehicle, etc.), inhome ac and dc loads, and power conversion circuits. The dc equipment is connected at a single dc bus. The power system is connected to the outside of the home. The connection is the two ways of electricity: one is direct to the in-home ac load, and the other is the interface circuit. We apply a technology of uni-directional, single-phase grid-connected 


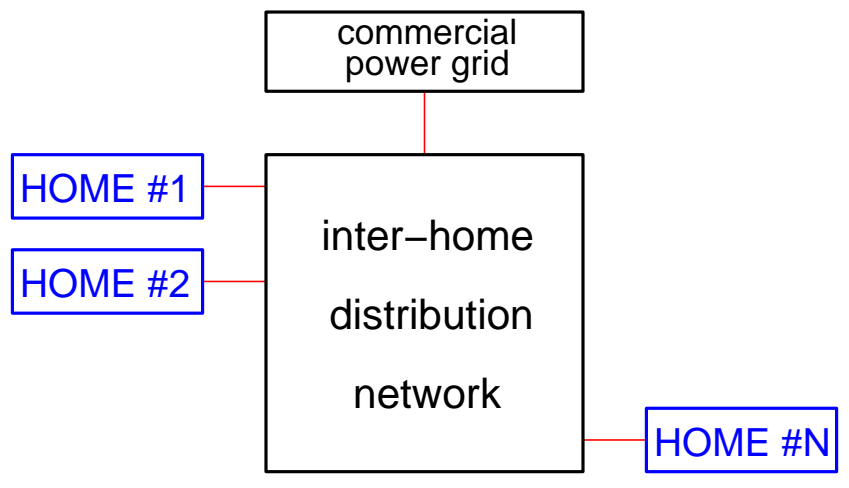

Fig. 3. Physical architecture of the electric power system with multiple homes. The $N$ homes are interconnected locally and connected via a lowvoltage distribution network and connected to the commercial power grid.

inverters to the interface circuit between the in-home dc system and the ac system. This technology is based on [5][7]. In [5], [6], the authors propose a new control method for dc/ac conversion (inverter) that connects a PV array with the commercial ac grid. The control method uses a VoltageControlled Oscillator (VCO) that adjusts the frequency of inverter output and achieves frequency synchronization. In [7] the authors propose the synchronous inverter and show that it enables the mutual synchronization of frequency as well as phase and the enhancement of stability in a gridconnected system. The interface circuit has main two roles for the control of electric-power: one is the exchange of power between the in-home dc system and the ac system, and the other is the enhancement of stability in the connected power system.

\section{Inter-home Architecture}

Figure 3 shows the physical architecture of the electric power system with multiple homes. The $N$ homes are interconnected locally and connected via a low-voltage distribution network. The whole network is connected to the commercial power grid. For the distribution network, there are several graph topologies on which the $N$ homes are dynamically interacted: ladder, ring, and star topologies. The choice of network topology affects the ability of electricpower management (controllability) as well as stability and reliability of the entire power system. In Section IV, we will numerically study dynamics of the power system with three $(N=3)$ homes and ladder topology.

\section{Mathematical Models}

In this section, we present mathematical models of the electric power system with $N$ homes. Each home includes a photovoltaic generation unit, a battery unit, an in-home dc load, and the interface circuit. This structure is a simplified one in Fig. 2. We replace the dc and ac loads by one dc load, which is valid for the ideal operation of power conversion circuit faced on the ac load. Before modeling in this section, we make the following assumptions:

1) Voltage amplitude is regulated at a nominal value.

2) In-home distribution network is lossless.

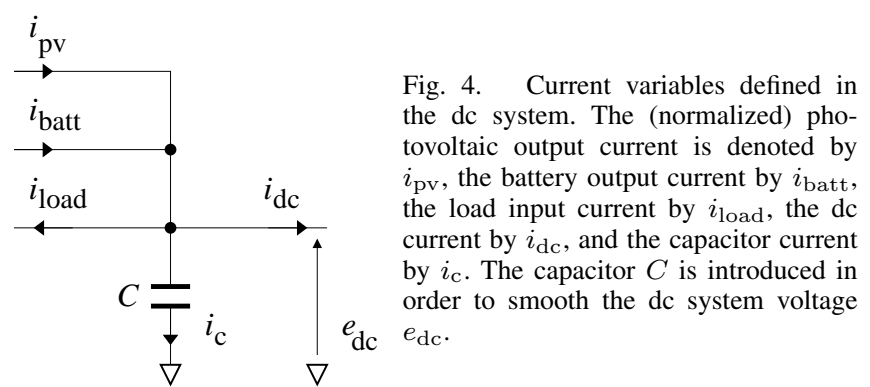

3) Interface circuit is lossless, and its operation is ideal.

4) Inter-home distribution network consists of passive elements.

5) Battery unit is not operated, and its dynamics are negligible.

6) Every home has common equipment of the in-home power system.

7) The commercial power grid is regarded as the infinite bus [8].

All of the assumptions except for 5) are reasonable for developing mathematical models of slow dynamics in the power system. The assumption 5) implies no battery control in the power system and may be sometimes crucial to the control objectives. In the current paper we address dynamics of the power system without control, and in a forthcoming paper we will present a mathematical model of the battery control. Table I is a list of variables and parameters that we use in this paper.

Here we provide the basic idea of mathematical modeling for the electric-power management. For each component in the homes, we provide a mathematical description of the relationship between the $\mathrm{dc}$ system voltage $e_{\mathrm{dc}}$ and an output current. Denote by $i_{\mathrm{pv}}, i_{\mathrm{batt}}, i_{\text {load }}$, and $i_{\mathrm{dc}}$ the (normalized) photovoltaic output current, the battery output current, the load input current, and the dc current: see Fig. 4. The capacitor current is also denoted by $i_{\mathrm{c}}$. In Fig. 4 the Kirchhoff's current law gives the following equality: at any moment $t$,

$$
i_{\mathrm{pv}}(t)+i_{\text {batt }}(t)=i_{\text {load }}(t)+i_{\mathrm{dc}}(t)+i_{\mathrm{c}}(t),
$$

where from the assumption 5),

$$
i_{\text {batt }}(t)=0 .
$$

The relationship between $e_{\mathrm{dc}}$ and $i_{\mathrm{c}}$ is simply the following:

$$
C \frac{\mathrm{d} e_{\mathrm{dc}}}{\mathrm{d} t}=i_{\mathrm{c}} .
$$

\section{A. Photovoltaic Generation Unit}

Figure 5 shows the simple circuit model of a PV cell that we use in this paper. This circuit model consists of the ideal dc current source $i_{\mathrm{ph}}$, the ideal diode, the shunt resistance $R_{\mathrm{sh}(\mathrm{pv})}$, and the series resistance $R_{\mathrm{s}(\mathrm{pv})}$. The parameter $i_{\mathrm{ph}}$ is the photoelectronic current produced in the cell and can change randomly due to the uncertain weather change. In 


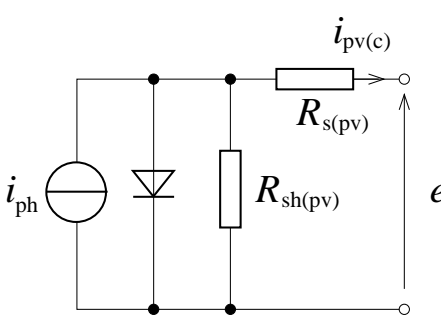

Fig. 5. Simple circuit model of a photovoltaic (PV) cell. This circuit model consists of the ideal current source $i_{\mathrm{ph}}$, the ideal diode, the shunt resistance $R_{\mathrm{sh}(\mathrm{pv})}$, and the se$e_{\mathrm{pv}(\mathrm{c})}$ ries resistance $R_{\mathrm{s}(\mathrm{pv})}$. The parameter $i_{\mathrm{ph}}$ is the photoelectronic current produced in the cell and can change randomly due to the uncertain weather change.

this model, we have the following relationship between the output current $i_{\mathrm{pv}(\mathrm{c})}$ and the cell terminal voltage $e_{\mathrm{pv}(\mathrm{c})}$ :

$$
\begin{aligned}
i_{\mathrm{ph}}= & I_{0}\left\{\exp \left(\frac{e_{\mathrm{pv}(\mathrm{c})}+i_{\mathrm{pv}(\mathrm{c})} R_{\mathrm{s}(\mathrm{pv})}}{V_{T}}\right)-1\right\} \\
& +\frac{e_{\mathrm{pv}(\mathrm{c})}+i_{\mathrm{pv}(\mathrm{c})} R_{\mathrm{s}(\mathrm{pv})}}{R_{\mathrm{sh}(\mathrm{pv})}}+i_{\mathrm{pv}(\mathrm{c})},
\end{aligned}
$$

where $I_{0}$ is the reverse bias saturation current, and $V_{T}$ is the thermal voltage at temperature $T$ in Kelvin. The effect of the series resistance $R_{\mathrm{s}(\mathrm{pv})}$ is negligible because its normal value is very small. Let us read $i_{\mathrm{pv}}$ as the output current of a PV array that consists of $N_{\mathrm{s}}$ cells in series and $N_{\mathrm{p}}$ cells in parallel. Thus $i_{\mathrm{pv}}$ is represented as

$$
\begin{aligned}
i_{\mathrm{pv}}= & N_{\mathrm{p}} i_{\mathrm{pv}(\mathrm{c})} \\
\sim & N_{\mathrm{p}}\left[i_{\mathrm{ph}}-I_{0}\left\{\exp \left(\frac{e_{\mathrm{dc}}}{N_{\mathrm{s}} V_{T}}\right)-1\right\}\right. \\
& \left.-\frac{e_{\mathrm{dc}}}{N_{\mathrm{s}} R_{\mathrm{sh}(\mathrm{pv})}}\right],
\end{aligned}
$$

where we used the direct relation $e_{\mathrm{pv}(\mathrm{c})}=e_{\mathrm{dc}} / N_{\mathrm{s}}$ under an additional assumption of the PV array in which each cell has a common value of terminal voltage. Note that the $\mathrm{dc} / \mathrm{dc}$ converter is normally introduced to adjust the output voltage $N_{\mathrm{s}} e_{\mathrm{pv}(\mathrm{c})}$ of the $\mathrm{PV}$ array. In the current modeling, we implicitly suppose that no dc/dc converter for the PV array is introduced, or a dc/dc converter with a constant conversion ratio $d_{\mathrm{pv}}$ is introduced. It is straightforward to add a controller model of $d_{\mathrm{pv}}$ to the current PV model in the averaging framework [15].

\section{B. In-home dc load}

Simply we consider the in-home load as a constant power load, which is denoted by $p_{\text {load }}$. Then the load input current $i_{\text {load }}$ is represented as

$$
i_{\text {load }}=\frac{p_{\text {load }}}{e_{\mathrm{dc}}} .
$$

The parameter $p_{\text {load }}$ can change due to the time-dependent, uncertain profile of power consumption in homes. In the profile, appliance loads are very discontinuous.

\section{Interface Circuit}

Basically, we use the mathematical model of the interface circuit presented in [5], [7], but improve it slightly in order to represent interaction dynamics of multiple homes. Fig. 6 shows the block diagram of the Voltage-Controlled

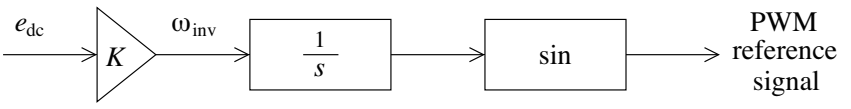

Fig. 6. Block diagram of the Voltage-Controlled Oscillator (VCO) that produces the PWM reference signal [7]. The VCO achieves frequency synchronization between different homes.

Oscillator (VCO) that produces the PWM reference signal. The mathematical model of the VCO is simply the following:

$$
\omega_{\mathrm{inv}}=\frac{\omega_{0}}{E_{0}} e_{\mathrm{dc}}=K\left(e_{\mathrm{dc}}-E_{0}\right)+\omega_{0},
$$

where $\omega_{0}$ is the nominal angular frequency $(2 \pi \times 50 \mathrm{~Hz}$ or $2 \pi \times 60 \mathrm{~Hz}), E_{0}$ is the nominal value of $e_{\mathrm{dc}}$, and $K:=\omega_{0} / E_{0}$ is the gain constant for frequency regulation. By defining the new variable $\Delta \omega$ as $\omega_{\text {inv }}-\omega_{0}$, we have

$$
\Delta \omega=K\left(e_{\mathrm{dc}}-E_{0}\right) .
$$

Here, by the assumption that the interface circuit is lossless, we have the following equality for active power balance that bridges between the dc and ac systems:

$$
e_{\mathrm{dc}} i_{\mathrm{dc}}=\operatorname{Re}\left[\dot{E} \dot{I}^{*}\right]=: p
$$

where we denote by $\dot{E}$ (or $\dot{I}$ ) the ac output voltage (or current) of the interface circuit in phasor, and the symbol * stands for the conjugate operation of complex variables. The variable $\delta$ is termed as the phase angle in literature of power systems engineering [8]. The instantaneous value of $\dot{E}$ corresponds to $e_{\mathrm{ac}}(t):=\sqrt{2}|\dot{E}| \sin \left(\omega_{0} t+\delta\right)$. With eqs. (1), (3), (8), and (9), we have the following differential equations that represent the time changes of $\delta$ and $\Delta \omega$ :

$$
\left.\begin{array}{rl}
\frac{\mathrm{d} \delta}{\mathrm{d} t} & =\Delta \omega \\
\frac{\mathrm{d} \Delta \omega}{\mathrm{d} t} & =\frac{K}{C}\left(i_{\mathrm{pv}}+i_{\mathrm{batt}}-i_{\mathrm{load}}-\frac{p}{e_{\mathrm{dc}}}\right),
\end{array}\right\}
$$

where recall that $e_{\mathrm{dc}}$ is a function of $\Delta \omega$. The term $p$ of the active power output from a single home is derived in the next subsection after modeling of the inter-home distribution network. We will see that $p$ becomes a function of phase angles of all homes operating in a power system.

\section{Inter-home Distribution Network}

In this modeling, we address slow dynamics of frequency and active power whose time scale is sufficiently large compared with the alternative signal (namely, $20 \mathrm{~ms}$ or $16.7 \mathrm{~ms}$ ). In this case, the inter-home distribution network can be modeled in a framework of quasi-static phasor representation. For given cutset matrix $C$ and branch admittance matrix $Y_{\text {branch }}$ of the distribution network, we derive the admittance matrix $Y$ as $C Y_{\text {branch }} C^{\top}$. The symbol $T$ stands for the transpose operation of vectors and matrices. Thus we have $\dot{I}=\mathrm{Y} \dot{\boldsymbol{V}}$, where $\dot{\boldsymbol{I}}:=\left(\dot{I}_{1}, \ldots, \dot{I}_{N}, \dot{I}_{N+1}\right)^{\top}$ is the output current vector of the $N$ interface circuits and the commercial grid $(N+1)$, and $\dot{\boldsymbol{V}}:=\left(\dot{V}_{1}, \ldots, \dot{V}_{N}, \dot{V}_{N+1}\right)^{\top}$ is the voltage vector at the linkage points of the $N$ interface circuits and the commercial grid. What we want now is the relation between $\dot{\boldsymbol{I}}$ and the 
output voltage vectors $\dot{\boldsymbol{E}}:=\left(\dot{E}_{1}, \ldots, \dot{E}_{N}, \dot{E}_{N+1}\right)^{\top}$ of the $N$ interface circuits and the commercial grid. Here let us define the vector of linkage impedance from each home to the network as $\dot{Z}_{\text {link. }}$ Then we have

$$
\dot{\boldsymbol{E}}=\operatorname{diag}\left(\dot{\boldsymbol{Z}}_{\text {link }}\right) \dot{\boldsymbol{I}}+\dot{\boldsymbol{V}} \text {. }
$$

By using $\dot{\boldsymbol{I}}=\mathrm{Y} \dot{\boldsymbol{V}}$ we obtain the relation between $\dot{\boldsymbol{I}}$ and $\dot{\boldsymbol{E}}$ as follows:

$$
\dot{\boldsymbol{I}}=(\mathrm{G}+\mathrm{jB}) \dot{\boldsymbol{E}},
$$

with

$$
\mathrm{G}+\mathrm{jB}:=\left(\mathrm{Y} \operatorname{diag}\left(\dot{\boldsymbol{Z}}_{\text {link }}\right)+\mathrm{I}_{N+1}\right)^{-1} \mathrm{Y},
$$

where $\mathrm{I}_{N+1}$ stands for the identity matrix of size $N+1$. Both $G$ and $B$ are real-valued, symmetric matrices with constant entries. The relation (12) is used for the calculation of the term $p_{k}$ of active output power from home $\# k$ $(k=1,2, \ldots, N)$ :

$$
\begin{aligned}
p_{k}:= & \operatorname{Re}\left[\dot{E}_{k} \dot{I}_{k}^{*}\right] \\
= & G_{k k} E_{k}^{2}+\sum_{l=1, l \neq k}^{N+1} E_{k} E_{l}\left\{G_{k l} \cos \left(\delta_{k}-\delta_{l}\right)\right. \\
& \left.+B_{k l} \sin \left(\delta_{k}-\delta_{l}\right)\right\},
\end{aligned}
$$

where $G_{k l}$ stands for the matrix entry in the $k$-th row and $l$-th column. This is why the term $p_{k}$ is a function of phase angles $\delta_{k}$ in all the homes.

\section{E. The Full Model}

As a result, we have the following full model that represents the dynamics of frequency and active power in the $N$ homes: for home $\# k(k=1, \ldots, N)$,

$$
\begin{aligned}
\frac{\mathrm{d} \delta_{k}}{\mathrm{~d} t}= & \Delta \omega_{k} \\
\frac{\mathrm{d} \Delta \omega_{k}}{\mathrm{~d} t}= & \frac{K}{C}\left(i_{\mathrm{pv}, k}-\frac{p_{\mathrm{load}, k}}{e_{\mathrm{dc}, k}}-\frac{p_{k}}{e_{\mathrm{dc}, k}}\right), \\
e_{\mathrm{dc}, k}= & E_{0}+\frac{\Delta \omega_{k}}{K}, \\
i_{\mathrm{pv}, k}= & N_{\mathrm{p}}\left[i_{\mathrm{ph}, k}-I_{0}\left\{\exp \left(\frac{e_{\mathrm{dc}, k}}{N_{\mathrm{s}} V_{T}}\right)-1\right\}\right. \\
& \left.-\frac{e_{\mathrm{dc}, k}}{N_{\mathrm{s}} R_{\mathrm{sh}(\mathrm{pv})}}\right],
\end{aligned}
$$

where the term $p_{k}$ of the active power output is presented in (14).

Because of the design of interface circuit, we show that the full model (15) has a coupling structure similar to that in the multi degree-of-freedom swing equations for transient stability analysis of multi-machine power grids [8]-[10]. Under the parameter setting in Sec. IV, the model (15) has a particular structure of strong neighbor coupling and weak long-range effect, which has been extensively studied in [10], [16]-[18]. However, compared with [16]-[18], there is one different feature of the model (15) in which the anti-symmetric coupling term, $G_{k l} \cos \left(\delta_{k}-\delta_{l}\right)$, exists. The term comes from a non-zero resistive element in the interhome distribution network, whose value is compatible with

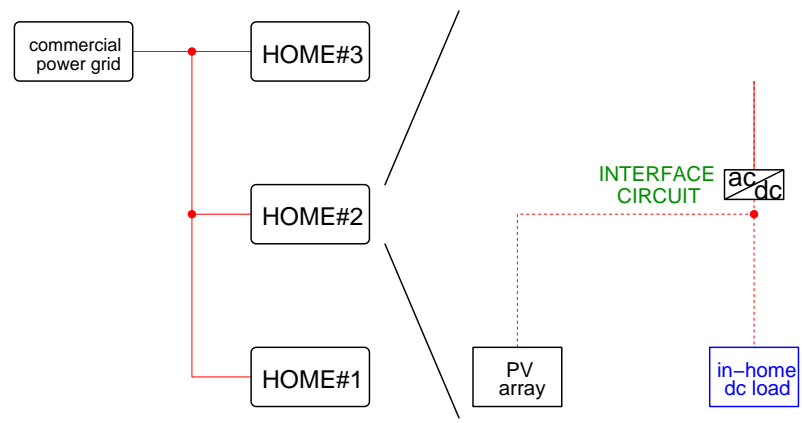

Fig. 7. Electric power system with three homes that is used for numerical simulations in this paper. The three homes are interconnected via a laddertype inter-home network. Each home has a simple structure of the PV array, the dc load, and the interface circuit.

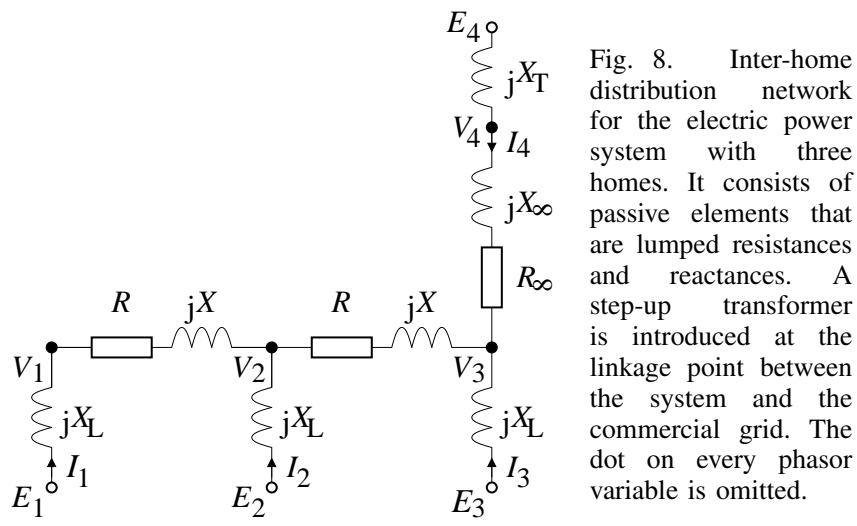

the reactance element in a low-voltage line: see Tab. I. In [19] the author proves that there is no energy function for a multi degree-of-freedom swing equation if its degree is more than two, and it has an anti-symmetric coupling term like above. The existence of energy function implies no (bounded) oscillatory attractor in the swing equation. In this way, we suggest that the slow dynamics described by the model (15) are likely non-integrable.

\section{NOMinal Dynamics}

In this section, we present an example of the slow dynamics in the case of three $(N=3)$ homes. The corresponding electric power system is shown in Fig. 7. Since the current model does not fully include control systems, the associated dynamics are regarded as an uncontrolled pure case. Understanding the pure case allows us to define nominal dynamics of the power system that are not only regulated by control but also used for the purpose of electric-power management. The developed model possesses many uncertain parameters that include the injection current of the PV array and the load profile. We numerically investigate the slow dynamics under a change of the parameter of the PV array. The setting of parameters for simulations is given in Tab. I.

\section{A. Modeling of a Ladder Network}

Before simulations we compute the conductance matrix $G$ and the susceptance matrix B. Consider the inter-home distribution network with the ladder topology shown in Fig. 8. The parameters $R, X, R_{\infty}, X_{\infty}, R_{\mathrm{L}}, X_{\mathrm{L}}$, and $X_{\mathrm{T}}$ 


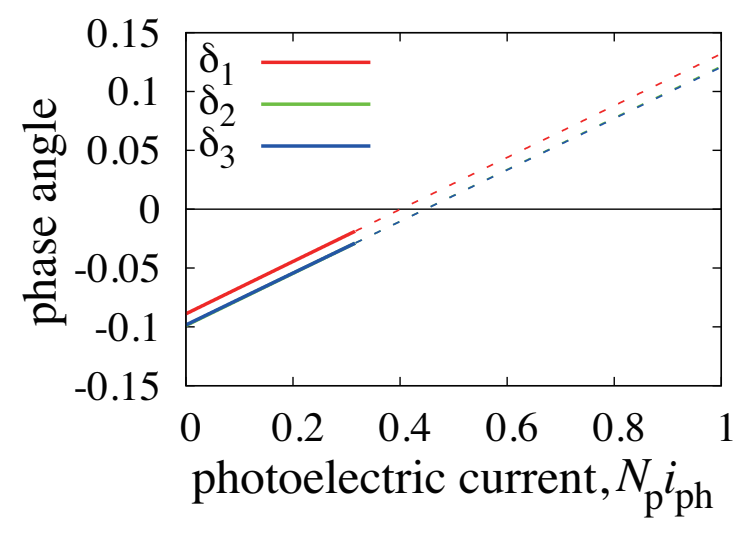

Fig. 9. Change of the values of the phase angles $\delta_{k}$ under an equilibrium of the dynamical system (15). The solid lines represent the stable equilibriums, and the dashed lines the unstable equilibriums. The values of $\delta_{k}$ monotonically increase as the photoelectric current $N_{\mathrm{p}} i_{\mathrm{ph}}$ increases. At $N_{\mathrm{p}} i_{\mathrm{ph}}=0.316$, the stability of the equilibrium changes due to the supercritical Hopf bifurcation.

are constant and given in Tab. I. For the network, the cutset matrix $C$, the branch admittance matrix $Y_{\text {branch }}$, and the linkage impedance vector $\dot{Z}_{\text {link }}$ are the following:

$$
\begin{aligned}
\mathrm{C}= & \left(\begin{array}{rrr}
1 & 0 & 0 \\
-1 & 1 & 0 \\
0 & -1 & 1 \\
0 & 0 & -1
\end{array}\right), \\
\mathrm{Y}_{\text {branch }}= & \operatorname{diag}\left(\left((R+\mathrm{j} X)^{-1},(R+\mathrm{j} X)^{-1},\right.\right. \\
& \left.\left.\left(R_{\infty}+\mathrm{j} X_{\infty}\right)^{-1}\right)^{\top}\right), \\
\mathrm{Y}= & \mathrm{CY}_{\text {branch }} \mathrm{C}^{\top}, \\
\dot{Z}_{\text {link }}= & \mathrm{j}\left(X_{\mathrm{L}}, X_{\mathrm{L}}, X_{\mathrm{L}}, X_{\mathrm{T}}\right)^{\top} .
\end{aligned}
$$

With these, the two matrices $G$ and $B$ are computed in (13): see Tab. II.

\section{B. Steady-state Characteristics}

First, we consider steady-state characteristics of the electric power system with the three homes. Fig. 9 shows the values of phase angles $\delta_{k}$ under an equilibrium with the change of the photoelectric current $N_{\mathrm{p}} i_{\mathrm{ph}}$. As presented in Tab. I, we consider the case of non-uniform load profile, $\left(p_{\text {load }, 1}, p_{\text {load }, 2}, p_{\text {load }, 3}\right)=(0,0.5,0.5)$. We also consider the uniform photoelectric current for every home, that is, $i_{\mathrm{ph}, k}=i_{\mathrm{ph}}$. This uniform setting of $i_{\mathrm{ph}}$ is valid if the $N$ homes are geographically close. The non-uniform setting of loads results in non-uniform equilibrium values of $\delta_{k}$ as shown in Fig. 9. In this figure, the solid lines represent the stable equilibriums, and the dashed lines the unstable equilibriums. Thus we see that the stability of the equilibrium changes at $N_{\mathrm{p}} i_{\mathrm{ph}}=0.316$. Based on the linearized system of (15) around the equilibrium, the change of stability is due to the supercritical Hopf bifurcation [20]. After the bifurcation value, a stable limit cycle is generated around the equilibrium. The stable limit cycle implies a undamped, sustained oscillation in frequency and active power, and it is regarded as a undesirable operating condition of the power system.
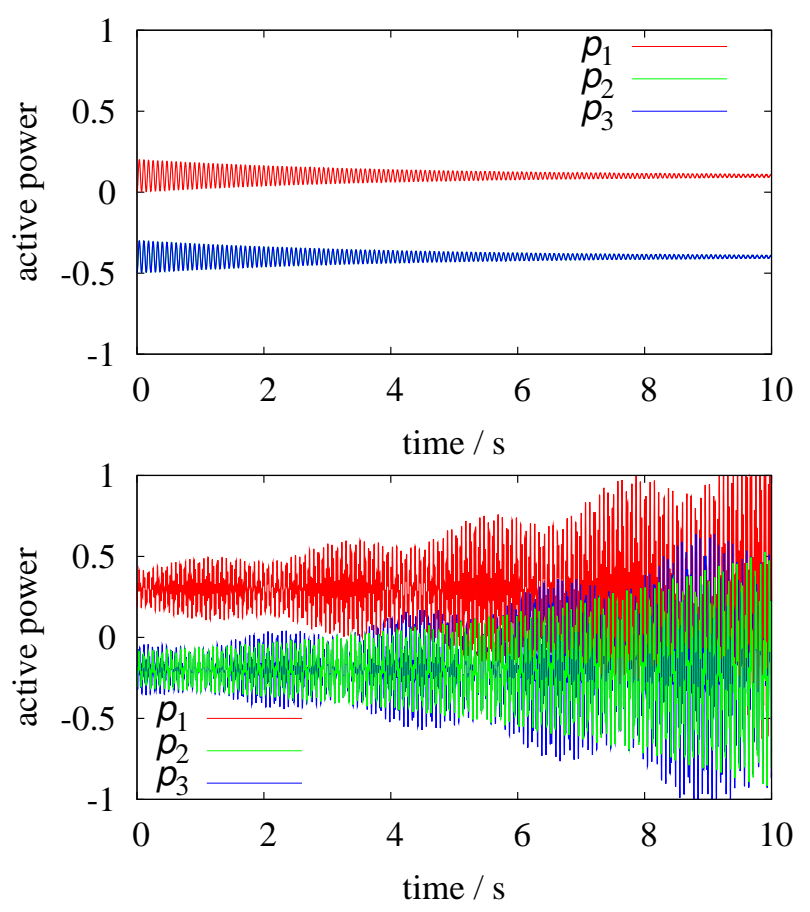

Fig. 10. Short-term step responses of the active power output $p_{k}$ when the photoelectric current $N_{\mathrm{p}} i_{\mathrm{ph}}$ suddenly changes at $t=0$. In the upper figure, $N_{\mathrm{p}} i_{\mathrm{ph}}$ changes from 0.1 to 0.2 , and in the lower figure it changes from 0.3 to 0.4 . The step response in the low figure grows gradually and finally reaches a undamped, sustained oscillation.

\section{Short-term and Long-term Responses}

Next, we focus on short-term dynamics caused by a change of the photoelectric current $N_{\mathrm{p}} i_{\mathrm{ph}}$. In the previous sub-section, it is shown that the stability of the equilibrium is lost due to the change of $N_{\mathrm{p}} i_{\mathrm{ph}}$. The parameter $N_{\mathrm{p}} i_{\mathrm{ph}}$ is governed by the uncertain weather condition and can change suddenly: see [21]. This implies a possibility of the destabilization of the steady operating condition of the power system. Fig. 10 shows short-term step responses of the active power output $p_{k}$ when $N_{\mathrm{p}} i_{\mathrm{ph}}$ suddenly changes at $t=0 \mathrm{~s}$. In the upper figure, $N_{\mathrm{p}} i_{\mathrm{ph}}$ changes from 0.1 to 0.2 , and in the lower figure it changes from 0.3 to 0.4 . The value 0.4 of the parameter $N_{\mathrm{p}} i_{\mathrm{ph}}$ is larger than the bifurcation value (0.316). Due to the nominal dynamics with the Hopf bifurcation, in the lower figure we observe that the step response grows gradually and finally reaches a sustained oscillation characterized by a stable limit cycle.

Finally, we discuss long-term dynamics caused by a combination of the bifurcation phenomenon and uncertain change of the photoelectric current. As mentioned above, the parameter $N_{\mathrm{p}} i_{\mathrm{ph}}$ is uncertain and can be modeled in a probabilistic way. One example of such time series of $N_{\mathrm{p}} i_{\mathrm{ph}}$ is shown in the upper figure of Fig. 11. The value of $N_{\mathrm{p}} i_{\mathrm{ph}}$ changes at every 5 seconds. The time series is generated as a Gaussian distribution with mean 0.25 and standard deviation 0.071 . The lower figure shows the probability density of the time series. The density is computed by counting the time spent for each $N_{\mathrm{p}} i_{\mathrm{ph}}$ and dividing it by the total 
time duration in Fig. 11. This time series is regarded as an uncertain input to the dynamical system (15). This viewpoint of the dynamical system comes from the idea of uncertainty propagation in nonlinear systems [22]. Fig. 12 shows the long-term responses of the active power output $p_{k}$ against the time series of $N_{\mathrm{p}} i_{\mathrm{ph}}$. We see intermittent behaviors in the course of long-term dynamics that do not appear in the time series of $N_{\mathrm{p}} i_{\mathrm{ph}}$. They result from a combination of the Hopf bifurcation and the uncertain input. While the value of $N_{\mathrm{p}} i_{\mathrm{ph}}$ is larger than the bifurcation value, the slow dynamics do not settle down to any equilibrium and sometimes grow (see the lower figure of Fig. 10). On the other hand, while $N_{\mathrm{p}} i_{\mathrm{ph}}$ is smaller than the bifurcation value, the dynamics tend to converge to a stable equilibrium (see the upper figure of Fig. 10). This data suggests a possibility of complex behaviors caused by a combination of uncertain renewables and nominal nonlinear dynamics of an electric power system.

\section{Conclusions}

We reported physical architectures and mathematical models for electric-power management of multiple homes. Numerical simulations of the models provided a part of nominal dynamics of the electric power system with three homes. Due to the supercritical Hopf bifurcation and associated sustained oscillations, we indicate that the nominal dynamics may induce a nonlinear propagation of the uncertain input of the PV array to the active output power of each home. Follow-up studies to these are as follows: (i) validation of the proposed models by real data acquisitions; (ii) further analysis of the nonlinear propagation using practical data such as in [21] and other uncertain parameters such as house loads; (iii) design of technical and dynamic operational architectures for our management goal; (iv) their theoretical analysis including automatic verification of stability [23]; and (v) mathematical modeling of battery control.

\section{ACKNOWLEDGEMENTS}

The authors are grateful to Dr. Ryo Takahashi for his valuable comments, Dr. Yuichi Yokoi, Mr. Tsuguhiro Takuno, and Mr. Masataka Minami for their careful reading of the manuscript. They also thank anonymous reviewers for their suggestive comments that we follow for revising the first version of the manuscript.

\section{REFERENCES}

[1] Report to NIST on the Smart Grid Interoperability Standards Roadmap (Contract No. SB1341-09-CN-0031), Prepared by the Electric Power Research Institute, June 17, 2009.

[2] http://www.net.ist.i.kyoto-u.ac.jp/ice-it (in Japanese).

[3] http://www.amsterdamsmartcity.com.

[4] The Influence of Architecture in Engineering Systems, in Engineering Systems Monograph, Prepared by the MIT Engineering Systems Division, March 2004 (available at http://esd.mit.edu/symposium/monograph/).

[5] K. Harada and K. Murata, "Interface circuit between solarcell and commercial ac bus," Transactions of the Institute for Engineers of Communications and Electronics, vol. J69-C, pp. 1458-1464, November 1986, (in Japanese).

[6] _ - "On the automatic interconnection between solar cell and ac power source," in Proceedings of the Telecommunications Energy Conference, New Orleans, USA, November 1984, pp. 259-262.
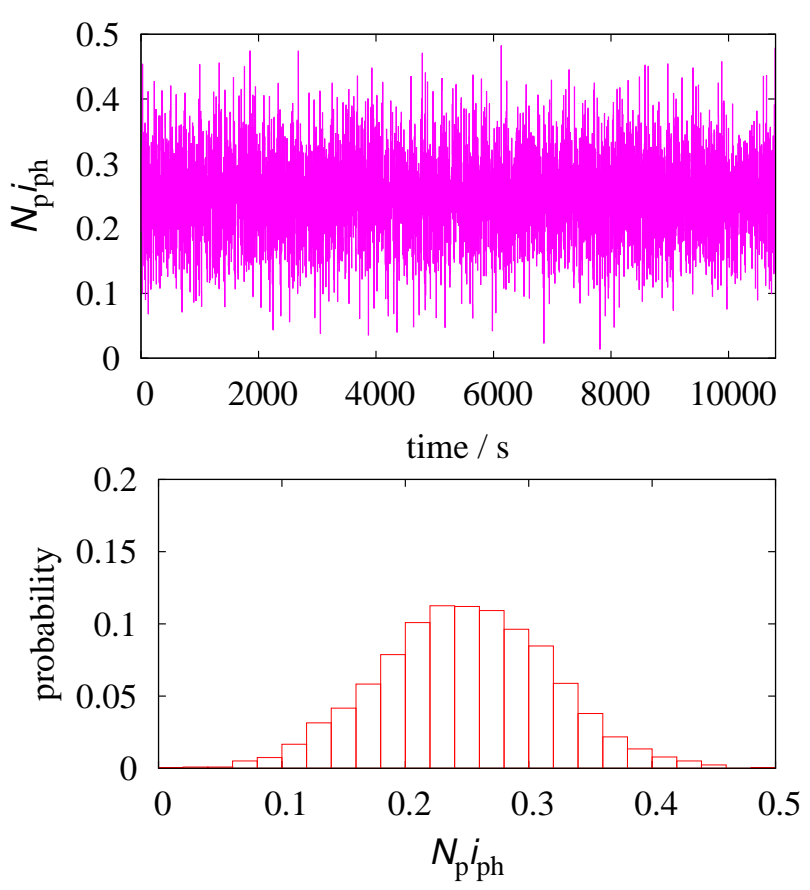

Fig. 11. One example of time series and probability density of uncertain input from the photoelectric current of the PV array. The time series is generated as a Gaussian distribution with mean 0.25 and standard deviation 0.071 .

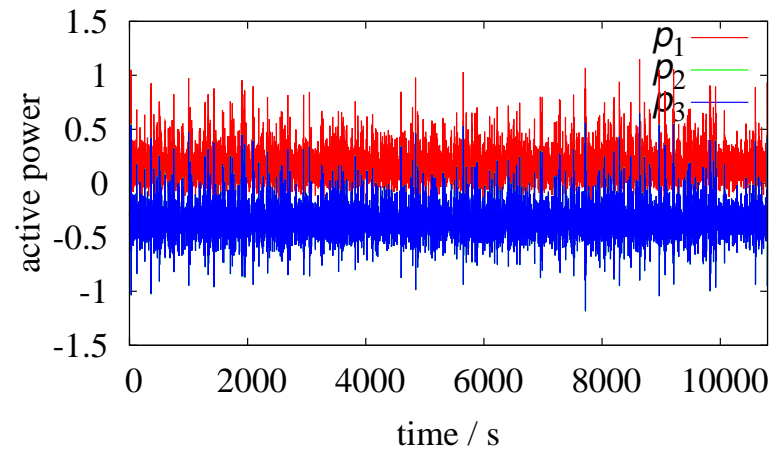

Fig. 12. Long-term responses of the active power output $p_{k}$ while the photoelectric current $N_{\mathrm{p}} i_{\mathrm{ph}}$ changes in a probabilistic way. The intermittent dynamics are caused by a combination of the Hopf bifurcation and the random change of $N_{\mathrm{p}} i_{\mathrm{ph}}$ shown in Fig. 11 .

[7] T. Hikihara, T. Sawada, and T. Funaki, "Enhanced entrainment of synchronous inverters for distributed power sources," IEICE Trans. Fund. Electr., vol. E90-A, no. 11, pp. 2516-2525, November 2007.

[8] P. Kundur, Power System Stability and Control. McGraw-Hill, 1994.

[9] H. D. Chiang, "Power system stability," in Wiley Encyclopedia of Electrical and Electronics Engineering, J. G. Webster, Ed. New York: John Wiley \& Sons, March 1999, pp. 105-137.

[10] Y. Susuki, I. Mezić, and T. Hikihara, "Coherent swing instability of power grids," J. Nonlinear Sci., vol. 21, no. 3, pp. 403-439, June 2011.

[11] IEEE/CIGRE Joint Task Force on Stability Terms and Definitions, "Definition and classification of power system stability," IEEE Trans. Power Syst., vol. 19, no. 2, pp. 1387-1401, May 2004.

[12] S. B. Kjaer, J. K. Pedersen, and F. Blaabjerg, "A review of single-phase grid connected inverters for photovoltaic modules," IEEE Trans. Ind. Applicat., vol. 41, pp. 1292-1306, September/October 2005.

[13] B. Lasseter, "Microgrids," in IEEE PES Winter Meeting Panel, Columbus, USA, 2001, pp. 146-149.

[14] N. Hatziargyriou, H. Asano, R. Iravani, and C. Marnay, "Microgrids," IEEE Power Energy Mag., vol. 5, no. 4, pp. 78-94, July/August 2007. 
TABLE I

LIST OF VARIABLES AND PARAMETERS OF THE MATHEMATICAL MODELS FOR THE ELECTRIC POWER SYSTEM WITH MULTIPLE HOMES

\begin{tabular}{|c|c|c|c|}
\hline Symbol & Meaning & $\begin{array}{l}\text { Value fixed } \\
\text { for simulation }\end{array}$ & $\begin{array}{c}\text { Normalized } \\
\text { Value }\end{array}$ \\
\hline $\begin{array}{l}e_{\mathrm{dc}, k} \\
i_{\mathrm{dc}, k}\end{array}$ & \multicolumn{3}{|l|}{$\begin{array}{l}\text { DC volage input to the interface circuit in home } \# k \\
\text { DC current input to the interface circuit in home } \# k\end{array}$} \\
\hline$e_{\mathrm{pv}(\mathrm{c}), k}$ & \multicolumn{3}{|l|}{ Voltage output of the PV cell in home \#k } \\
\hline$i_{\mathrm{pv}(\mathrm{c}), k}$ & \multicolumn{3}{|l|}{ Current output of the PV cell in home \#k } \\
\hline$i_{\mathrm{pv}, k}$ & \multicolumn{3}{|l|}{ Current output of the PV array in home $\# k$} \\
\hline$R_{\mathrm{s}(\mathrm{pv})}$ & Internal series resistance in the PV cell model & $0 \Omega$ & 0 \\
\hline$R_{\mathrm{sh}(\mathrm{pv})}$ & Internal shunt resistance in the PV cell model & $10 \Omega$ & 0.439 \\
\hline $\begin{array}{l}i_{\mathrm{ph}, k} \\
I_{0}\end{array}$ & \multicolumn{2}{|l|}{ Photoelectric (solar-induced) current of the PV cell model in home $\# k$} & $6.17 \times 10^{-17}$ \\
\hline$q$ & Elementary charge & \multicolumn{2}{|c|}{$1.602176487(40) \times 10^{-19} \mathrm{C}$} \\
\hline$k_{\mathrm{B}}$ & Boltzmann constant & \multicolumn{2}{|c|}{$1.3806504(24) \times 10^{-23} \mathrm{~J} / \mathrm{K}$} \\
\hline$T$ & Temperature (in Kelvin) & $300 \mathrm{~K}$ & \\
\hline$V_{T}$ & Thermal voltage $k_{\mathrm{B}} T / q$ & $0.0258 \mathrm{~V}$ & \multirow{2}{*}{$6.97 \times 10^{-5}$} \\
\hline$N_{\mathrm{s}}$ & Number of the PV cells connected in a series way & 480 & \\
\hline$N_{\mathrm{p}}$ & Number of the PV cells connected in a parallel way & 20 & \\
\hline$i_{\text {batt }}$ & \multicolumn{2}{|l|}{ Current flowing from/to the battery } & 0 \\
\hline$i_{\text {load }, k}$ & \multicolumn{3}{|l|}{ Current input to the load in home $\# k$} \\
\hline$p_{\text {load }, k}$ & \multicolumn{2}{|l|}{ Power consumed in the load of home \#k } & $\begin{array}{l}0.0(k=1) \\
0.5(k=2) \\
0.5(k=3)\end{array}$ \\
\hline$\delta_{k}$ & \multirow{2}{*}{\multicolumn{3}{|c|}{$\begin{array}{l}\text { Phase angle of the interface circuit in home } \# k \\
\text { Phase angle of the commercial power grid (infinite bus) }\end{array}$}} \\
\hline$\delta_{0}$ & & & 0 \\
\hline$\omega_{\text {inv }, k}$ & \multicolumn{3}{|l|}{ Angular frequency of the PWM reference signal in home $\# k$} \\
\hline & Nominal angular frequency & $2 \pi \times 60 \mathrm{~Hz}$ & 1 \\
\hline$\Delta \omega_{k}$ & \multicolumn{3}{|l|}{ Derivation of the angular frequency $\omega_{\mathrm{inv}, k}$ relative to $\omega_{0}$} \\
\hline$E_{0}$ & Nominal value of the dc system voltage $e_{\mathrm{dc}, k}$ in home $\# k$ & \multirow{2}{*}{$\begin{array}{c}370 \mathrm{~V} \\
1.109 \mathrm{rad} / \mathrm{s} / \mathrm{V}\end{array}$} & 1 \\
\hline$K$ & Gain constant of the Voltage-Controlled Oscillator (VCO), defined as $\omega_{0} / E_{0}$ & & 1 \\
\hline$C$ & Smoothing capacitance in the interface circuit & $10 \mathrm{mF}$ & 85.95 \\
\hline$i_{\mathrm{c}, k}$ & \multicolumn{3}{|l|}{ Capacitor Current of the interface circuit in home \#k } \\
\hline$p_{k}$ & \multicolumn{3}{|l|}{ Active power output of the interface circuit in home $\# k$} \\
\hline $\mathrm{C}$ & \multicolumn{3}{|l|}{ Cutset matrix of the inter-home distribution network } \\
\hline Ybranch & \multicolumn{3}{|l|}{ Branch admittance matrix of the inter-home distribution network } \\
\hline & \multicolumn{3}{|l|}{ Admittance matrix of the inter-home distribution network, given as $\mathrm{CY}_{\mathrm{branch}} \mathrm{C}^{\top}$} \\
\hline$\dot{V} ; \dot{V}_{k}$ & \multicolumn{3}{|l|}{ Voltages vector at $N$ linkage points; Voltage at the linkage point to home \#k } \\
\hline$\dot{\boldsymbol{E}} ; \dot{E}_{k}$ & \multicolumn{3}{|c|}{ Voltages vector at the interface circuits for $N$ homes; Output voltage of the interface circuit at home \#k } \\
\hline$\dot{\boldsymbol{I}} ; \dot{I}_{k}$ & \multicolumn{3}{|c|}{ Currents vector at the interface circuits for $N$ homes; Output current of the interface circuit at home $\# k$} \\
\hline$\dot{Z}_{\text {link }}$ & Linkage impedance vector for $N$ homes & & \\
\hline G & Conductance matrix for the network-reduced model, defined in (13) & & \\
\hline B & Susceptance matrix for the network-reduced model, defined in (13) & & \\
\hline$R+\mathrm{j} X$ & Line impedance between nearest neighbor homes & $3.0 \times 10^{-3} \Omega(R)$ & $4.5 \times 10^{-4}$ \\
\hline & & $4.0 \times 10^{-3} \Omega(X)$ & $6.0 \times 10^{-4}$ \\
\hline$R_{\infty}+\mathrm{j} X_{\infty}$ & Line impedance connected to the commercial power grid & $3.0 \times 10^{-2} \Omega\left(R_{\infty}\right)$ & $4.5 \times 10^{-3}$ \\
\hline & & $4.0 \times 10^{-2} \Omega\left(X_{\infty}\right)$ & $6.0 \times 10^{-3}$ \\
\hline$X_{\mathrm{T}}$ & Transformer reactance & & 0.06 \\
\hline$X_{\mathrm{L}}$ & Linkage reactance between a home and the distribution network & & 0.02 \\
\hline
\end{tabular}

TABLE II

VALUES OF THE REAL-VALUED MATRICES G AND B

\begin{tabular}{crrrr}
\hline$G_{k l} / B_{k l}$ & $l=1$ & $l=2$ & $l=3$ & $l=4$ \\
\hline$k=1$ & $0.7638 /$ & $0.0239 / 15.063$ & $-0.3764 / 14.480$ & $-0.4113 / 4.356$ \\
$k=2$ & & $0.3635 /$ & $-0.0619 / 14.923$ & $-0.3256 / 4.500$ \\
$k=3$ & & & $0.5866 /$ & $-0.1483 / 4.782$ \\
$k=4$ & & & & $0.8852 /$ \\
\hline
\end{tabular}

[15] J. G. Kassakian, M. F. Schlecht, and G. C. Verghese, Principles of Power Electronics. Addison-Wesley, 1991.

[16] I. Mezić, "On the dynamics of molecular conformation," Proc. Natl. Acad. Sci. USA, vol. 103, no. 20, pp. 7542-7547, 2006.

[17] P. Du Toit, I. Mezić, and J. Marsden, "Coupled oscillator models with no scale separation," Physica D, vol. 238, no. 5, pp. 490-501, March 2009.

[18] B. Eisenhower and I. Mezić, "Targeted activation in deterministic and stochastic systems," Phys. Rev. E, vol. 81, p. 026603, February 2010.

[19] H. D. Chiang, "Study of the existence of energy functions for power systems with losses," IEEE Trans. Circuits Syst., vol. 36, no. 11, pp. 1423-1429, November 1989.

[20] J. Guckenheimer and P. Holmes, Nonlinear Oscillations, Dynamical
Systems, and Bifurcations of Vector Fields, ser. Applied Mathematical Sciences. New York: Springer-Verlag, 1983, vol. 42.

[21] N. Jenkins, R. Allan, P. Crossley, D. Kirschen, and G. Strbac, Embedded Generation, ser. IET Power and Energy Series. London: The Institute of Engineering and Technology, 2000, vol. 31 .

[22] I. Mezić and T. Runolfsson, "Uncertainty propagation in dynamical systems," Automatica, vol. 44, pp. 3003-3013, November 2006.

[23] Y. Susuki, T. J. Koo, H. Ebina, T. Yamazaki, T. Ochi, T. Uemura, and T. Hikihara, "A hybrid system approach to the analysis and design of power grid dynamic performance," Proc. IEEE, vol. 99, 2011, (to appear). 